\title{
ON THE RADIUS OF CONVEXITY AND STARLIKENESS OF UNIVALENT FUNCTIONS
}

\author{
S. K. BAJPAI AND R. S. L. SRIVASTAVA
}

\begin{abstract}
In this paper, the converses of the theorems of Bernardi (Trans. Amer. Math. Soc. 135 (1969), 429-446) for the subclasses of univalent functions, namely, starlike functions of order $\beta$, convex functions of order $\beta$ and close-to-convex functions of type $\beta$ and order $\lambda$ have been derived. In particular, these results are sharp and contain the theorems of Padmanabhan (J. London Math. Soc. (2) 1 (1969), 226-231) and Bernardi (Proc. Amer. Math. Soc. 24 (1970), 312-318) as special cases.
\end{abstract}

1. Introduction. In this paper we study some classes of univalent functions. By $S$ we denote the class of functions $w=f(z)=z+\sum_{n=2}^{\infty} \mathrm{a}_{n} z^{n}$, which are regular and univalent in the unit disc $D\{|z|<1\}$, while $S^{*}$ denotes the class of functions in $S$ which map $D$ onto a starlike region with respect to the origin. An equivalent analytic characterization for functions of $S^{*}$ is well known [8]. By $S_{\beta}^{*}$ we denote the class of functions $f(z)$ in $S^{*}$ having the additional property

$$
\operatorname{Re}\left\{\frac{z f^{\prime}(z)}{f(z)}\right\} \geqq \beta ; \quad z \in D ; \quad 0 \leqq \beta \leqq 1 .
$$

Here $\beta$ is referred as the order of starlike functions $f(z)$ and we identify $S_{0}^{*} \equiv S^{*}$. The class of functions $f(z)$, which are in $S$ and map $D$ onto a convex domain, is denoted by $C$, while $C_{\beta}$ denotes the class of univalent functions of order $\beta$, if

$$
\operatorname{Re}\left\{1+\frac{z f^{\prime \prime}(z)}{f^{\prime}(z)}\right\} \geqq \beta ; \quad z \in D, \quad 0 \leqq \beta \leqq 1 .
$$

If $f(z) \in S$ and $g(z) \in S_{\beta}^{*}$ satisfy the condition

$$
\operatorname{Re}\left\{\frac{z f^{\prime}(z)}{g(z)}\right\} \geqq \lambda ; \quad z \in D, \quad 0 \leqq \lambda \leqq 1,
$$

Presented to the Society, June 15, 1970; received by the editors March 30, 1971. AMS 1969 subject classifications. Primary 3042.

Key words and phrases. Radius of convexity, convexity and starlikeness of univalent functions.

(C) American Mathematical Society 1972 
then $f(z)$ is said to be a close-to-convex function of order $\lambda$ and type $\beta$. We denote this class by $\Gamma(\lambda, \beta)$. If $\lambda=0=\beta$, then $f(z)$ is simply said to be a close-to-convex function with respect to the function $g(z)$ and the class $\Gamma(0,0)$ is identified by $\Gamma$. This concept of close-to-convex functions is due to Kaplan [3] and its extension appears in the works of Robertson, Libera and others.

Libera [4] in 1965 established the following theorems:

TheOREM A [LIBERA]. If $f \in S^{*}$ (or $\left.f \in C\right)$ then the function $F(z)=$ $(2 / z) \int_{0}^{z} f(t) d t \in S^{*}$ (or $\left.F \in C\right)$.

THEOREM B [LIBERA]. If $f \in \Gamma$ with respect to $g(z)$ and $F(z)=(2 / z) \int_{0}^{z} f(t) d t$ and $G(z)=(2 / z) \int_{0}^{z} g(t) d t$ then $F \in \Gamma$ with respect to $G$.

Bernardi [1] extended Theorems A and B, and proved the following:

TheOREM C [Bernardi]. If $f(z) \in S^{*}$ (or $\left.C\right), c=1,2,3, \cdots$,

$$
g(z)=\sum_{n=1}^{\infty}\left(\frac{c+1}{c+n}\right) a_{n} z^{n}=(c+1) z^{-c} \int_{0}^{z} t^{c-1} f(t) d t \quad\left(a_{1}=1\right)
$$

then $g(z) \in S^{*}$ (or $\left.C\right)$.

THEOREM D [BeRNARDI]. If $f \in \Gamma$ with respect to $g$ and

$$
F(z)=(c+1) z^{-c} \int_{0}^{z} t^{c-1} f(t) d t ; \quad G(z)=(c+1) z^{-c} \int_{0}^{z} t^{c-1} g(t) d t,
$$

$c=1,2,3, \cdots$, then $F \in \Gamma$ with respect to $G$.

The converse problem of Libera [4] was treated by Livingston [5] who proved the following:

TheOREm E [Livingston]. If $F \in S^{*}$ then $f(z)=\frac{1}{2}[z F(z)]^{\prime}$ is starlike for $|z|<\frac{1}{2}$. This result is sharp.

Theorem F [Livingston]. If $F$ is in $C$, then $f(z)=\frac{1}{2}[z F(z)]^{\prime}$ is univalent in $D$ and is convex for $|z|<\frac{1}{2}$. This result is sharp.

Bernardi [2] again considered the converse problem of Theorems $\mathrm{C}$ and $\mathrm{D}$ and obtained the following:

THEOREM G [BERNARDI]. If $f(z)=z+\sum_{n=2}^{\infty} a_{n} z^{n}$,

$$
g(z)=\sum_{n=1}^{\infty}\left(\frac{c+1}{c+n}\right) a_{n} z^{n}=(c+1) z^{-c} \int_{0}^{z} t^{c-1} f(t) d t
$$

with $a_{1}=1$ and $c=1,2,3, \cdots$ and $g(z) \in S^{*}$ (or $C$ ) then $f(z)$ is starlike (or convex) in the region $|z|<\left(-2+\left(3+c^{2}\right)^{1 / 2}\right) /(c-1)$ for $c=2,3,4, \cdots$ and $|z|<\frac{1}{2}$ for $c=1$. This result is sharp. 
THEOREM H [BERNARDI]. Let $F(z)$ be close-to-convex with respect to $G(z) \in S^{*}$ and

$$
f(z)=\frac{1}{1+c} z^{1-c}\left[z^{c} F(z)\right]^{\prime}, \quad g(z)=\frac{1}{1+c} z^{1-c}\left[z^{c} G(z)\right]^{\prime}
$$

then $f(z)$ is close-to-convex with respect to $g(z)$ in the region

$$
|z|<\left(-2+\left(3+c^{2}\right)^{1 / 2}\right) /(c-1)
$$

for $c=2,3,4, \cdots$ and $|z|<\frac{1}{2}$ if $c=1$.

Padmanabhan [7] considered the converse problem of Libera [4] for the class $S_{\beta}^{*}, C_{\beta}$ and $\Gamma(\lambda, \beta)$, where $0 \leqq \beta \leqq \frac{1}{2}$. In this paper we are mainly concerned with radius of starlikeness and radius of convexity for functions in $S_{\beta}^{*}, C_{\beta}$ and $\Gamma(\lambda, \beta)$, respectively. In particular, we derive the converses of Theorems $\mathrm{G}$ and $\mathrm{H}$ of Bernardi for the classes $S_{\beta}^{*}, \Gamma(\lambda, \beta)$ and $C_{\beta}$. We notice that these results are sharp. With these extensions we deduce the theorems of Padmanabhan [7] also. Incidently, the proof of our Theorem 1, which includes a theorem of Padmanabhan, is much simpler and can also be adopted for the restricted case considered by him. We state here a lemma due to Bernardi [1] which we shall need,

LEMMA [1, p. 430]. Let $f(z)$ and $g(z)$ be regular in $|z|<1, g(z)$ map $|z|<1$ onto a many sheeted starlike region, $\alpha, \beta$ real,

$$
\operatorname{Re}\left\{e^{i \beta} \frac{f^{\prime}(z)}{g^{\prime}(z)}\right\}>\alpha
$$

for $|z|<1, g(0)=f(0)=0$. Then $\operatorname{Re}\left\{e^{i \beta} f(z) / g(z)\right\}>\alpha$ for $|z|<1$.

Further, if $f(z) \in S^{*}, g(z)=\int_{0}^{z} H(t) d t=\int_{0}^{z} t^{p-1} f(t) d t$, then $g(z)$ is $(p+1)$ valent starlike for $p=1,2,3, \cdots$.

2. We shall prove the following:

THEOREM 1. If $f(z)=z+\sum_{n=2}^{\infty} a_{n} z^{n} \in S_{\beta}^{*}$ and

$$
g(z)=\sum_{n=1}^{\infty}\left(\frac{c+1}{c+n}\right) a_{n} z^{n}=(c+1) z^{-c} \int_{0}^{z} t^{c-1} f(t) d,
$$

with $a_{1}=1$ and $c=1,2,3, \cdots$, then $g(z) \in S_{\beta}^{*}$ and conversely, if $g(z) \in S_{\beta}^{*}$ then $f(z)$ is starlike of order $\beta$ in the region

$$
\begin{aligned}
|z|<r_{0} & =\frac{-(2-\beta)+\left(3+\beta^{2}+c^{2}+2 c \beta-2 \beta\right)^{1 / 2}}{c+2 \beta-1}
\end{aligned}
$$


Proof. If $J(z)=\int_{0}^{z} t^{c-1} f(t) d t$ then it easily follows on lines similar to those given by Bernardi $[1$, p. 431] that

$$
\operatorname{Re}\left\{\frac{\left[z^{c+1} g^{\prime}(z)\right]^{\prime}}{J^{\prime}(z)}\right\}=(c+1) \operatorname{Re}\left\{\frac{z f^{\prime}(z)}{f(z)}\right\} \geqq(c+1) \beta .
$$

From (2.1) and the lemma, the first part of the theorem follows.

Conversely, by the hypothesis of the theorem we have

$$
\frac{z g^{\prime}(z)}{g(z)}=\frac{z J^{\prime}-c J}{J} .
$$

Further, since $g(z)$ is starlike function of order $\beta$, so there exists a function $\omega(z)$ which is regular in the unit disc $D$ and satisfies the conditions of Schwarz's lemma, such that

$$
\frac{z g^{\prime}(z)}{g(z)}=\frac{1-(1-2 \beta) \omega(z)}{1+\omega(z)} .
$$

From (2.2) and (2.3) it follows that

$$
f(z)=\frac{[(1+c)+\{c+(2 \beta-1)\} \omega(z)] J}{[1+\omega(z)] z^{c}} .
$$

Differentiating $f(z)$ logarithmically and simplifying finally we get

$$
\begin{aligned}
\frac{z f^{\prime}(z)}{f(z)} & -\beta=(1-\beta) \\
\times & {\left[\frac{1-\omega(z)}{1+\omega(z)}-\frac{2 z \omega^{\prime}(z)}{[1+\omega(z)][(1+c)+(c+2 \beta-1) \omega(z)]}\right] }
\end{aligned}
$$

But

and

$$
\operatorname{Re}\left\{\frac{1-\omega(z)}{1+\omega(z)}\right\}=\frac{1-|\omega(z)|^{2}}{|1+\omega(z)|^{2}}
$$

$$
\begin{aligned}
& \operatorname{Re}\left\{\frac{2 z \omega^{\prime}(z)}{[1+\omega(z)][(1+c)+(c+2 \beta-1) \omega(z)]}\right\} \\
& \leqq \frac{2|z|\left(1-|\omega(z)|^{2}\right)}{\left(1-|z|^{2}\right)|1+\omega(z)||(1+c)+(c+2 \beta-1) \omega(z)|}
\end{aligned}
$$

The last inequality is obtained by using the following well-known inequality [6, p. 168]:

$$
\left|\omega^{\prime}(z)\right| \leqq\left(1-|\omega(z)|^{2}\right) /\left(1-|z|^{2}\right)
$$


From (2.4) thru (2.7), we note that $f(z)$ is starlike of order $\beta$, if

$$
\frac{2|z|\left(1-|\omega(z)|^{2}\right)}{|1+\omega(z)|\left(1-|z|^{2}\right)|(1+c)+(c+2 \beta-1) \omega(z)|} \leqq \frac{1-|\omega(z)|^{2}}{|1+\omega(z)|^{2}}
$$

or

$$
\frac{2|z|}{1-|z|^{2}} \leqq(1+c)\left|1+\frac{c+2 \beta-1}{1+c} \omega(z)\right| /|1+\omega(z)| .
$$

Since $|\omega(z)| \leqq|z|$ and $(c+2 \beta-1) /(1+c) \leqq 1$, we have

$$
1+\frac{c+2 \beta-1}{1+c}|z| /(1+|z|) \leqq\left|1+\frac{c+2 \beta-1}{1+c} \omega(z)\right| /|1+\omega(z)| .
$$

Hence, by (2.8) and (2.9), we obtain that $f(z) \in S_{\beta}^{*}$ if

i.e., if

$$
2|z| \leqq\{(1+c)+(c+2 \beta-1)|z|\}(1-|z|)
$$

$$
(1+c)-2(2-\beta)|z|-(c+2 \beta-1)|z|^{2}>0 .
$$

Let $P(|z|)=P(r)=(1+c)-2(2-\beta) r-(c+2 \beta-1) r^{2}$. Since $P(0)=1+c$ and $P^{\prime}(r)<0$, the positive root $r_{0}$ for which $P(r)>0$ must be less than the root of the polynomial $P(r)=0$. This gives the required value of $r_{0}$ and the proof of Theorem 1 is complete.

The following example shows that the result of Theorem 1 is sharp for each $c$.

EXAMPle 1. Consider the function

$$
g(z)=z(1-z)^{-2(1-\beta)} ; \quad 0 \leqq \beta \leqq 1 .
$$

Clearly $g(z) \in S_{\beta}^{*}$ and $f(z)=\left(z^{1-c} /(1+c)\right)\left[z^{c} g(z)\right]^{\prime}$ imply that

$$
\frac{z f^{\prime}(z)}{f(z)}-\beta=\frac{(1-\beta)\left[(1+c)+2(2-\beta) z-(c+2 \beta-1) z^{2}\right]}{(1-z)[(1+c)-(c+2 \beta-1) z]} .
$$

Thus $z f^{\prime}(z) / f(z)-\beta=0$ for $z=-r_{0}$. Hence $f(z)$ is not starlike in any disc $|z|<r$, if $r>r_{0}$.

Theorem $\mathrm{G}$ of Bernardi now follows by taking $c=1,2,3, \cdots$ and $\beta=0$.

If we take $c=1$ and $0 \leqq \beta \leqq \frac{1}{2}$, then the following theorem of Padmanabhan [7] follows as a corollary to Theorem 1 .

Theorem [PadmanABhan]. Let $g(z) \in S_{\beta}^{*}$. Then $f(z)=\frac{1}{2}[z g(z)]^{\prime}$ is starlike of order $\beta$ for

$$
|z|<\frac{(\beta-2)+\left(\beta^{2}+4\right)^{1 / 2}}{2 \beta} ; \quad 0 \leqq \beta \leqq \frac{1}{2} .
$$


THEOREM 2. If $g(z) \in C_{\beta}, f(z)=(1 /(1+c)) z^{1-c}\left[z^{c} g(z)\right]^{\prime}, c=1,2,3, \cdots$, then $f(z)$ is convex of order $\beta$ in $|z|<r_{0}$ where $r_{0}$ is defined as in Theorem 1 . The result is sharp.

Proof. Proof of Theorem 2 follows immediately by using the fact that if $g(z) \in C_{\beta}$ then $z g^{\prime}(z) \in S_{\beta}^{*}$ and conversely (see [8]).

The following example shows that the result of Theorem 2 is sharp.

EXAMPLE 2. Let $g(z)=1-(1-z)^{2 \beta-1} /(2 \beta-1) ; \beta \neq \frac{1}{2}$ and if $\beta=\frac{1}{2}$ then $g(z)=-\log (1-z)$.

If $\beta \neq \frac{1}{2}$ then by direct computation we find that

$$
\begin{aligned}
f(z) & =\frac{(2 \beta-1) c-c(1-z)^{2 \beta-1}+(2 \beta-1) z(1-z)^{2 \beta-2}}{(1+c)(2 \beta-1)}, \\
f^{\prime}(z) & =\frac{(1-z)^{2 \beta-3}}{1+c}[(1+c)+(1-c-2 \beta) z],
\end{aligned}
$$

and, therefore

$$
(1-\beta)+\frac{z f^{\prime \prime}(z)}{f^{\prime}(z)}=\frac{(1-\beta)\left[(1+c)+2(2-\beta) z-(c+2 \beta-1) z^{2}\right]}{(1-z)[(1+c)-(c+2 \beta-1) z]} .
$$

Thus the expression $(1-\beta)+z f^{\prime \prime}(z) / f^{\prime}(z)$ vanishes for $z=-r_{0}$, hence $f(z)$ is not convex of order $\beta$ in any disc $|z|<r, r>r_{0}$. Similarly for $\beta=\frac{1}{2}$ and $g(z)=-\log (1-z)$, sharpness of the theorem can be established.

If we take $c=1$ and $0 \leqq \beta \leqq \frac{1}{2}$ in Theorem 2 , then the following theorem of Padmanabhan [7] is obtained as a corollary to Theorem 2.

Theorem [Padmanabhan]. Let $g(z) \in C_{\beta}$. Then $f(z)=\frac{1}{2}[\operatorname{zg}(z)]^{\prime} \in C_{\beta}$ for

$$
|z|<\left[\frac{(\beta-2)+\left(\beta^{2}+4\right)^{1 / 2}}{2 \beta}\right] \text {. }
$$

THEOREM 3. Let $f(z)=\left(z^{1-c} /(1+c)\right)\left[z^{c} F(z)\right]^{\prime}$ and $g(z)=\left(z^{1-c} /(1+c)\right) \times$ $\left[z^{c} G(z)\right]^{\prime}, c=1,2,3, \cdots, G(z) \in S_{\beta}^{*}$ and $F(z) \in \Gamma(\lambda, \beta)$ with respect to $G(z)$. Then $f(z) \in \Gamma(\lambda, \beta)$ with respect to the function $g(z) \in S_{\beta}^{*}$ for $|z|<r_{0}$. The result is sharp.

Proof. Since $G(z) \in S_{\beta}^{*}$, from Theorem 1 , we have

$$
\operatorname{Re}\left\{\frac{z g^{\prime}(z)}{g(z)}\right\} \geqq \beta \quad \text { for }|z|<r_{0} .
$$

Also, since $F(z) \in \Gamma(\lambda, \beta)$ with respect to $G(z)$, there exists an analytic function $\omega(z)$ satisfying the conditions of Schwarz's lemma such that

$$
P(z) \equiv \frac{z F^{\prime}(z)}{G(z)}=\frac{1-(1-2 \lambda) \omega(z)}{1+\omega(z)} .
$$


But $P(z)$ can also be written as

$$
P(z) \int_{0}^{z} t^{c-1} g(t) d t=z^{c} f(z)-c \int_{0}^{z} t^{c-1} f(t) d t .
$$

By differentiating (2.10) with respect to $z$, we obtain

$$
\frac{z f^{\prime}(z)}{g(z)}=P(z)+\frac{P^{\prime}(z)}{g(z)} z^{1-c} \int_{0}^{z} t^{c-1} g(t) d t .
$$

But

$$
\frac{1}{z^{c} g(z)} \int_{0}^{z} t^{c-1} g(t) d t=\frac{G(z)}{c G(z)+z G^{\prime}(z)} .
$$

Further, since $G(z) \in S_{\beta}^{*}$, there exists an analytic function $V(z)$ satisfying the conditions of Schwarz's lemma, such that

$$
\frac{z G^{\prime}(z)}{G(z)}=\frac{1-(1-2 \beta) V(z)}{1+V(z)} .
$$

From (2.13) we have

$$
\left[c+\frac{z G^{\prime}(z)}{G(z)}\right]^{-1}=\left[\frac{(c+1)+(c-1+2 \beta) V(z)}{1+V(z)}\right]^{-1} .
$$

Hence we have from (2.12) and (2.14) that

$$
\left[\int_{0}^{z} t^{c-1} g(t) d t\right] / z^{c} g(z)=\left[\frac{(c+1)+(c-1+2 \beta) V(z)}{1+V(z)}\right]^{-1}
$$

Thus by using (2.15) we obtain from (2.11) that

$$
\begin{aligned}
\operatorname{Re}\left\{\frac{z f^{\prime}(z)}{g(z)}\right\}-\lambda & \geqq \operatorname{Re}\{P(z)-\lambda\} \\
& \times\left[\frac{(c+1)-2(2-\beta)|z|-(c+2 \beta-1)|z|^{2}}{(1-|z|)[(c+1)+(c+2 \beta-1)|z|]}\right] .
\end{aligned}
$$

The inequality (2.16) implies that $f(z) \in \Gamma(\lambda, \beta)$ with respect to the function $g(z)$ if $|z|<r_{0}$. This completes the proof of Theorem 3. Sharpness of the theorem follows from Theorem 2 .

As a corollary to Theorem 3, Theorem $\mathrm{H}$ of Bernardi follows by taking $\beta=0$.

THEOREM 4. Let $F(z)=z+\sum_{n=2}^{\infty} a_{n} z^{n}$ be regular and have the property $\operatorname{Re}\left\{F^{\prime}(z)\right\}>\beta$ for $|z|<1, f(z)=(1 /(1+c)) z^{1-c}\left[z^{c} F(z)\right]^{\prime}, c=1,2,3, \cdots$. Then $\operatorname{Re}\left\{f^{\prime}(z)\right\}>\beta$ for $|z|<r_{1}=\left[-1+\left(2+2 c+c^{2}\right)^{1 / 2} / 1+c\right]$. The result is sharp. 
Proof. The proof given by Bernardi for theorem [2, p. 317] remains valid except for the following change.

$$
(1+c) \operatorname{Re}\left\{f^{\prime}(z)-\beta\right\} \geqq \operatorname{Re}\{P(z)-\beta\}\left[(1+c)-\frac{2|z|}{1-|z|^{2}}\right] .
$$

This result is sharp as is seen by the example,

$$
F(z)=(2 \beta-1) z-2(1-\beta) \log (1-z) .
$$

\section{REFERENCES}

1. S. D. Bernardi, Convex and starlike univalent functions, Trans. Amer. Math. Soc. i35 (1969), 429-446. MR 38 \#1243.

2. - The radius of univalence of certain analytic functions, Proc. Amer. Math. Soc. 24 (1970), 312-318. MR 40 \#4433.

3. W. Kaplan, Close-to-convex schlicht functions, Michigan Math. J. 1 (1952), 169-185. MR 14, 966.

4. R. J. Libera, Some classes of regular univalent functions, Proc. Amer. Math. Soc. 16 (1965), 755-758. MR 31 \#2389.

5. A. E. Livingston, On the radius of univalence of certain analytic functions, Proc. Amer. Math. Soc. 17 (1966), 352-357. MR 32 \#5861.

6. Z. Nehari, Conformal mapping, McGraw-Hill, New York, 1952. MR 13, 640.

7. K. S. Padmanabhan, On the radius of univalence of certain classes of analytic functions, J. London Math. Soc. (2) 1 (1969), 225-231. MR 40 \#331.

8. M. S. Robertson, The theory of univalent functions, Ann. of Math. 37 (1936), 374 408.

Department of Mathematics, Indian Institute of Technology, KanPur, India 